\title{
Kombination von Therapieprinzipien braucht sorgfältige Nutzen-Risiko-Abwägung
}

Die endokrine Therapie des fortgeschrittenen Hormonrezeptor(HR)-positiven Mammakarzinoms ist relativ gut verträglich, allerdings bei verschiedenen molekularen Subtypen unterschiedlich effektiv. Geprüft wurde daher die Kombination mit der Angiogenesehemmung.

n einer offenen Phase-III-Studie wurden Wirksamkeit und Sicherheit der Kombination aus Letrozol $(2,5 \mathrm{mg} / \mathrm{d}$ oral) plus dem VEGF(„vascular endothelial growth factor")-Hemmer Bevacizumab (15 mg/kg i.v., q3w) mit alleinigem Letrozol in der Erstlinientherapie von Frauen mit metastasiertem, HR-positivem Brustkrebs verglichen. Die Studie war so gepowert, dass eine $50 \%$ ige Verlängerung des medianen progressionsfreien Überlebens (PFS) von 6 auf 9 Monate nachgewiesen werden konnte. Daten von 343 Patientinnen (Alter: median 58 Jahre) standen für die Auswertung zur Verfügung.

Nach median 39 Monaten war das Progressionsrisiko unter der Kombinationstherapie gegenüber der Monotherapie signifikant geringer (Hazard Ratio
[HR] 0,75; $\mathrm{p}=0,016)$. Der Unterschied im medianen PFS war mit 4,6 Monaten geringer als erhofft (20,2 Monate unter der Kombination vs. 15,6 Monate unter Letrozol-Monotherapie); jedoch war das mediane PFS in beiden Gruppen deutlich länger als in der ursprünglichen Studienplanung angenommen. Nach 12 Monaten waren noch $73 \%$ der Patientinnen im Kombinationsarm und $61 \%$ der Patienten im Monotherapiearm progressionsfrei. Ein signifikanter Unterschied im Gesamtüberleben (OS) ergab sich nicht, das mediane OS lag im LetrozolArm bei 43,9, im Kombinationsarm bei 47,2 Monaten (HR 0,87; p = 0,188). Mindestens 1 unerwünschtes Ereignis vom Grad $\geq 3$ erlitten $47 \%$ der Kombinationsund $14 \%$ der Monotherapiegruppe. Am deutlichsten waren bei Kombinationsbe-

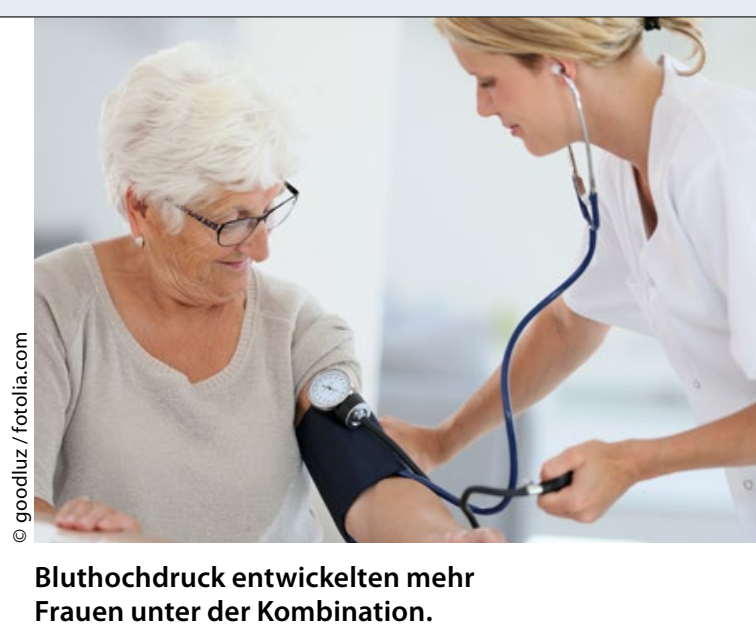

handlung Bluthochdruck (24 vs. $2 \%$ ) und Proteinurie (11 vs. $0 \%$ ) vermehrt.

Fazit: Letrozol plus Bevacizumab kann das PFS bei Frauen mit HR-positivem, metastasiertem Brustkrebs verlängern, erhöht aber auch die Rate an Grad-3/4Nebenwirkungen. Wichtig wären prädiktive Marker für Ansprechen und Toxizitiät, um Bevacizumab spezifischer einsetzen zu können. Friederike Klein

Dickler MN et al. Phase III Trial Evaluating Letrozole As First-Line Endocrine Therapy With or Without Bevacizumab for the Treatment of Postmenopausal Women With Hormone ReceptorPositive Advanced-Stage Breast Cancer: CALGB 40503 (Alliance). J Clin Oncol. 2016;34(22):2602-9.

\section{Langzeitdaten zu Tamoxifen bei prämenopausalem Mammakarzinom}

1984 starte die Phase-III-Studie „SBII:2pre“. Prämenopausale Brustkrebspatientinnen, die adjuvant 2 Jahre Tamoxifen erhalten hatten, lebten in dieser Studie länger progressionsfrei. Nun zeigen die Langzeitergebnisse auch einen Effekt auf das Überleben bei Patientinnen mit ER-positivem Tumor.

n der Studie hatten 564 prämenopausale Patientinnen randomisert entweder 2 Jahre Tamoxifen adjuvant oder keine weitere systemische Therapie erhalten. Nun wurden die Sterbedaten und Todesursachen bis zum Jahr 2014 analysiert. Ausgewertet wurden die kumulative Mortalität insgesamt (CM) und die kumulative brustkrebsabhängige Mortalität (CBCM). Nach median 26,3 Jahren waren von den 314 Todesfällen 262 auf die Brustkrebserkrankung zurückzuführen. Unabhängig vom Rezeptorstatus zeigte sich für die CM (Hazard Ratio [HR] 0,82; $\mathrm{p}=0,080)$ und für die CBCM (HR 0,81; $\mathrm{p}=0,090)$ ein Trend $\mathrm{zu}$ einem positiven Effekt der Tamoxifen-Therapie. Bei den 362 Frauen mit Östrogenrezeptor (ER)positivem Tumor war die Einnahme von Tamoxifen ebenfalls mit einer marginalen Reduktion in der CM assoziiert (HR 0,77; $\mathrm{p}=0,075)$. Die CBMC war aber signifikant verringert (HR 0,73; $\mathrm{p}=0,046)$.

Der Effekt der Tamoxifen-Therapie auf die Mortalität variierte im Verlauf. Für die CM lag die HR in den ersten 5 Jahren bei 1,05 , für die Jahre $>5$ bis 15 bei 0,58 (95\%-Konfidenzintervall [95\%-KI] 0,37$0,91)$ und danach bei 0,82 (95\%-KI 0,481,42). Die entsprechenden HR für die
CBCM betrugen 1,09 (95\%-KI 0,65-1,82), 0,53 (95\%-KI 0,33-0,86) und 0,72 (95\%-KI 0,36-1,44). Die meisten Patientinnen hatten keine adjuvante Chemotherapie erhalten. Die brustkrebsbedingte Übersterblichkeit der ER-positiven Patientinnen in den ersten Jahren dürfte heute durch moderne adjuvante Chemotherapie deutlich geringer ausfallen.

Fazit: Eine adjuvante 2-jährige Tamoxifen-Therapie führt bei prämenopausalen Patientinnen mit ER-positivem Bruskrebs langfristig zu einem Überlebensvorteil.

Friederike Klein

Ekholm M et al. Two Years of Adjuvant Tamoxifen Provides a Survival Benefit Compared With No Systemic Treatment in Premenopausal Patients With Primary Breast Cancer: Long-Term FollowUp (> 25 years) of the Phase III SBII:2pre Trial. J Clin Oncol. 2016;34(19):2232-8. 